Sandra Mabel Sánchez Sánchez

Investigação dos efeitos moleculares e celulares de variantes no gene RELN identificadas em um paciente com Transtorno do Espectro Autista

Investigation of the cellular and molecular effects of RELN gene variants in one patient with Autism Spectrum Disorder

São Paulo 
Sandra Mabel Sánchez Sánchez

\section{Investigação dos efeitos moleculares e celulares de variantes no gene RELN identificadas em um paciente com Transtorno do Espectro Autista}

Investigation of the cellular and molecular effects of RELN gene variants in one patient with Autism Spectrum Disorder

Dissertação apresentada ao Instituto
de Biociências da Universidade de
São Paulo, para a obtenção de
Título de mestre em Genética e
Biologia Evolutiva, na área de
Biologia/Genética.

Orientador (a): Dra. Andréa Laurato Sertié

São Paulo 


\section{Ficha Catalográfica}

Sánchez-Sánchez, Sandra
Investigação dos efeitos moleculares e
celulares de variantes no gene $R E L N$ identificadas em um
paciente com Transtorno do Espectro Autista.
Número de páginas:
Dissertação (Mestrado) - Instituto de
Biociências da Universidade de São Paulo. Departamento
de Genética e Biologia Evolutiva
1.Transtorno do espectro autista 2. Relina 3. Vias de
sinalização 4. Variantes raras
I. Universidade de São Paulo. Instituto de
Biociências. Departamento de Genética e Biologia
Evolutiva.

Prof(a). Dr(a).

Prof(a). Dr(a).

Prof(a). Dr(a).

Prof(a). Dr(a).

Dra. Andréa Laurato Sertié

Orientador(a) 


\section{Agradecimentos}

Toda conquista na vida do ser humano entrelaça uma arquitetura complexa na qual muitas energias participam. Todo homem humilde sabe que ele alcança o topo com a ajuda de muitos e a inspiração de muitos outros, mas, acima de tudo, todas as conquistas da vida devem ser agradecidas. É por isso que sou muito grata, em primeiro lugar, a Deus, porque me levando em suas mãos, permitiu que o plano perfeito que ele tem para mim fosse cumprido nesta fase da minha vida. "Tudo tem seu tempo", o tempo de Deus é diferente do dos homens, mas claramente é perfeito.

Sou grata também pela oportunidade que me dá ao poder compartilhar minhas realizações com pessoas tão especiais: minha família, o motor da minha vida, aqueles que me transmitiram responsabilidade e perseverança e cujas palavras de encorajamento e orações sempre estiveram no momento certo. Minhas amigas Jennifer, Marcela e Carolina por seu apoio incondicional, motivação e por serem minha família aqui no Brasil. Sabrina, Diana, Fernanda, Simone, Aline, Sheyla e Victoria por sua compreensão e motivação nos altos e baixos que nos acompanham diariamente, às vezes até os seus silêncios foram prova da sua companhia. Quero dar graças especiais a duas pessoas que admiro por seus grandes conhecimentos e humildade: Juliana, que chegou à minha vida em uma etapa crucial, obrigada pela ajuda, confiança, por sempre acreditar em mim e minhas capacidades, aprendi muito da vida com você, e, Mariana pela companhia, explicações, boa vontade, preocupação, alegria e amizade, obrigada por toda sua ajuda. Agradeço também ao André por sua ajuda e a todos os que de alguma forma contribuíram na realização de alguma etapa do desenvolvimento e finalização deste trabalho. Finalmente, agradeço a todos os estudantes de pós-graduação do IIEP, que com uma palavra, um sorriso e sua amabilidade fizeram acolhedor o laboratório, que pouco a pouco virou meu segundo lar. Estas manifestações de firmeza e carinho de todos e cada um sempre permaneceram no meu coração, tomara que de alguma maneira possa retribuir o tempo, esforço e dedicação que me brindaram.

Agradeço enormemente à Dra. Andréa Sertié, minha orientadora, pela paciência, compreensão, disposição, por ser sempre uma orientadora presente. Obrigada por todos os conhecimentos compartilhados, pelas exigências e por sempre se interessar em que eu aprendesse e fosse uma estudante melhor. Agradeço a oportunidade porque mesmo sem me conhecer acreditou que tinha potencial para ser sua aluna. 
Agradeço também a Dra. Anna Carla, uma pessoa muito humana, por sua ajuda, sabedoria e conselhos. Ao Instituto de Ensino e Pesquisa do Hospital Albert Einstein (IIEP) por suas instalações e equipamentos onde consegui realizar todos os experimentos e à Martinha técnico de laboratório por toda ajuda recebida tanto professional como pessoalmente; ao Instituto de Biociências da Universidade de São Paulo e ao CNPq pelo financiamento outorgado, porque graças a esse apoio consegui obter uma pós-graduação da melhor universidade do Brasil e conhecer outra cultura.

Não foi um caminho fácil de percorrer, coloquei todo o meu esforço, dedicação e compromisso; superei minhas próprias limitações e vivi minhas próprias vicissitudes. Graças a todos que estiveram perto de alguma ou outra forma e aos que ficaram ao meu lado. Graças também a vida que me ensinou o que sou capaz e que não há nada que não possa ser alcançado com perseverança e disciplina. A pessoa que volta para a Colômbia não é a mesma que veio, nem profissional nem pessoalmente.

Obrigada 
Índice

Capítulo 1- INTRODUÇÃO GERAL E OBJETIVOS ..............................................

Capítulo 2 - MATERIAIS E MÉTODOS................................................................... 15

Capítulo 3 - ARTIGO CIENTíFICO....................................................................20

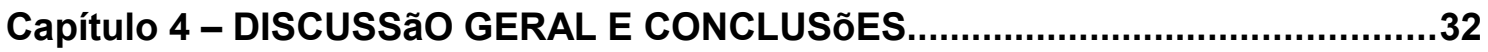

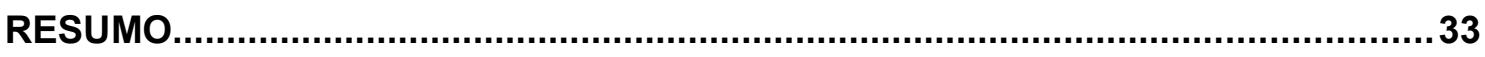

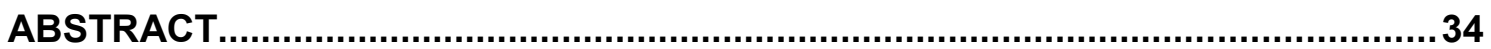

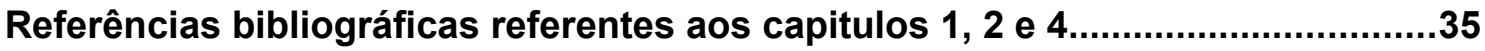




\section{CAPÍTULO 1- INTRODUÇÃO GERAL E OBJETIVOS}




\section{CAPÍTULO 1- INTRODUÇÃO GERAL E OBJETIVOS}

\subsection{Transtorno do espectro do autismo: sintomatologia e epidemiologia}

O transtorno do espectro do autismo (TEA) constitui um grupo heterogêneo de doenças do neurodesenvolvimento que tem seu início dentro dos primeiros anos de vida e é caracterizado principalmente por limitações na interação social e na comunicação verbal, bem como por comportamentos e interesses estereotipados e repetitivos [1]. Os sintomas e o grau de severidade variam amplamente entre os pacientes e outras manifestações clínicas podem estar presentes, tais como deficiências intelectual e motora, epilepsia, transtornos de ansiedade, problemas gastrointestinais, macrocefalia, dentre outros [2-4]. Cerca de $70 \%$ dos indivíduos com TEA apresentam comorbidades. Assim sendo, o diagnóstico pode ser difícil em decorrência da continua variabilidade no grau de comprometimento dos pacientes, da existência de comorbidades e da sobreposição dos sintomas [1].

Na edição mais recente do Manual Diagnóstico e Estatístico dos Transtornos mentais (DSM-5) da Associação Americana de Psiquiatria (2013), foram unificados sob o termo TEA vários transtornos que eram diagnosticados de forma independente. Logo, categorias como transtorno do autismo clássico, síndrome de Asperger, transtorno desintegrativo da infância e transtorno global do desenvolvimento sem outra especificação deixaram de existir como diagnósticos separados [1].

Estudos epidemiológicos estimam que a prevalência dos TEA na população mundial é de 1 para 68 indivíduos [5], sendo que esses transtornos são mais frequentes em homens do que em mulheres, em uma proporção de 4:1 [6,7]. No Brasil, a prevalência do TEA foi estimada em $0,27 \%$, valor abaixo do encontrado para a população mundial, o que foi atribuído à pequena amostra estudada, critérios diagnósticos inapropriados e deficiência nos registros dos casos [8]. 


\subsection{Transtorno do espectro do autismo: arquitetura genética e mecanismos etiológicos}

Embora as causas do TEA não sejam completamente esclarecidas, sabe-se que existe um forte componente genético em sua etiologia. A concordância estimada do TEA entre gêmeos monozigóticos é de aproximadamente $90 \%$ e entre gêmeos dizigóticos de aproximadamente $30 \%$. Ainda, sabe-se que quanto maior a porcentagem do genoma compartilhado com um individuo com TEA, maior o risco da doença para futuros familiares [9-12]. Com base nos estudos de concordância entre gêmeos e de recorrência familial, as estimativas de herdabilidade do TEA têm variado de $50-90 \%[10,13]$.

Cerca de $5-10 \%$ dos casos de TEA estão associados a síndromes monogênicas bem caracterizadas clinica e geneticamente e são comumente referidos como "TEA sindrômico". Dentre essas síndromes com alta prevalência de TEA no quadro clínico encontram-se, por exemplo, a síndrome do cromossomo $X$ frágil (gene FMR1), o complexo esclerose tuberosa (genes TSC1 e TSC2), a síndrome de Angelman (gene $U B E 3 A$ ), a neurofibromatose tipo 1 (gene NF1), as síndromes associadas a PTEN, a síndrome de RETT (gene MECP2 principalmente) e a síndrome de Timothy (gene CACNA1C) [14].

Os casos de TEA não associados a essas síndromes monogênicas são comumente referidos na literatura como "TEA não-sindrômico". Com o desenvolvimento das metodologias de análise genômica em larga escala, como a hibridização genômica comparativa (do inglês, comparative genomic hibridization CGH) e o sequenciamento de nova geração de exomas e genomas completos, tem sido possível a identificação em cerca de 15\%-20\% dos casos de TEA não-sindrômico de diversas variações do número de cópias de segmentos genômicos (do inglês, copy number variantions - CNVs), que podem envolver um ou mais genes [15-18], e de variantes pontuais não sinônimas em diversos genes candidatos, sendo a maior parte delas muito rara, isto é, presente em apenas um ou poucos pacientes $[19,20]$. Atualmente, mais de $2000 \mathrm{CNV}$ s e pelo menos 600 genes candidatos ao TEA estão descritos, de acordo com a mais recente atualização do banco de dados SFARI (Simons Foundation Autism Research Initiative) [21]. Embora para grande parte dessas variantes ainda não existam estudos que explorem os mecanismos patofisiológicos pelos quais elas estão envolvidas em TEA, esses achados revelam a ampla heterogeneidade genética que caracteriza esse transtorno. 
A arquitetura genética do TEA parece envolver variantes de novo e herdadas, tanto raras como comuns, que interagem de diferentes formas contribuindo para a etiologia da doença e ajustando-se em diversos modelos de herança. Assim, para cerca de $20-30 \%$ dos casos, o TEA é resultado de uma variante rara de novo e com alto impacto deletério em um gene principal (modelo monogênico) [19,22], ou é resultado de variantes raras e de impacto deletério moderado em dois ou poucos genes (modelo oligogênico ou multi-hit) [23-25]. O restante dos casos (aproximadamente $70-80 \%$ ) parece ser compatível com um modelo multifatorial de herança, no qual o fenótipo depende da interação de variantes mais frequentes e de efeito patogênico mais baixo em diversos genes e de fatores ambientais de risco (Figura 1) $[10,26]$.

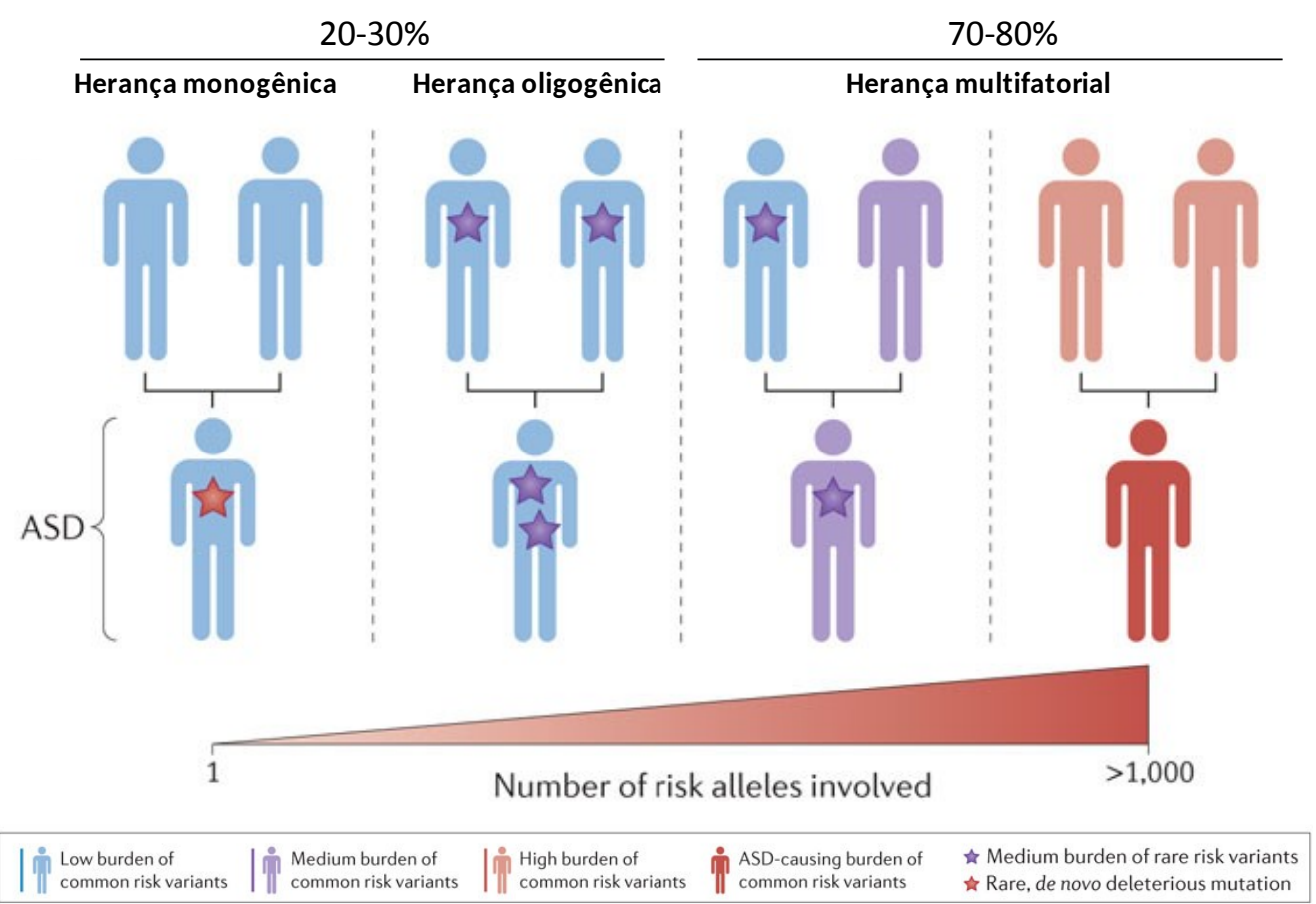

Figura 1. Interação entre variantes raras e comuns (background genético) na etiologia do TEA. A etiologia do TEA pode ocorrer por meio de vários caminhos. Uma variante deletéria altamente penetrante de novo pode causar TEA, mesmo em indivíduos com alto background genético atenuador, ou seja, com baixa carga de variantes de risco comuns (extrema esquerda). O TEA também pode surgir em indivíduos quando ambos os genitores forem portadores de uma carga média de variantes de risco raras (lado esquerdo do meio), ou se um dos genitores tiver uma carga média de variantes de risco raras e o outro genitor tiver uma carga média de variantes de risco comuns (lado direito do meio). Finalmente, as crianças podem desenvolver TEA se ambos os genitores forem portadores de uma grande quantidade de variantes de risco baixo comuns que se combinam no descendente (extrema direita) (Modificado de Bourgeron, 2015 [6]).

Segundo modelo proposto recentemente, a combinação de variantes raras de maior risco e alelos de baixo risco mais frequentes na população, como os polimorfismos de nucleotídeos únicos (do inglês, single nucleotide polymorphisms - 
SNPs), contribuem para moldar o fenótipo do TEA. Segundo esse modelo, dependendo da capacidade dos SNPs protetores de baixo risco em atenuar (tamponar) o impacto de variantes deletérias raras de mais alta penetrância, um indivíduo pode ou não desenvolver TEA $[6,27]$. Esse mecanismo parece contribuir para a grande diversidade fenotípica observada entre os pacientes e para a falta de penetrância de algumas variantes [6,28,29].

Estudos de ontologia genética mostram que grande parte dos genes com variantes deletérias comprovadamente associadas ao TEA atuam em vias biológicas envolvidas, sobretudo, com funções sinápticas, axonogênese e desenvolvimento neuronal, organização do citoesqueleto, adesão celular, migração neuronal, remodelamento da cromatina, proliferação celular e síntese proteica [6,30-32]. Embora esses estudos têm permitido um grande avanço no conhecimento dos mecanismos biológicos associados ao TEA, ainda existe muito a ser explorado e compreendido. Como acima comentado, a maior parte dos genes portadores de variantes candidatas ainda não foi estudada funcionalmente e seu envolvimento em TEA permanece especulativo. Portanto, o grande desafio atual no estudo da genética dos TEA é compreender como os genes com variantes de risco se relacionam e convergem em vias biológicas alteradas associadas ao transtorno, e quantas variantes patogênicas são necessárias para a penetrância completa da doença em cada paciente.

Dentre os genes de risco para TEA encontra-se RELN, foco do presente estudo. O gene RELN codifica Relina, uma grande glicoproteína secretada que controla a migração e o posicionamento dos neurônios, a formação e arborização de neuritos e o funcionamento das sinapses em várias regiões do encéfalo, tanto durante o desenvolvimento embrionário como na vida adulta [33-36].

\subsection{Migração neuronal e via de sinalização Relina-Dab1}

Estudos recentes, tanto neuroanatômicos como moleculares, têm sugerido que anormalidades na citoarquitetura do córtex cerebral, devido provavelmente a alterações na migração e na conectividade de células neurais durante o desenvolvimento, possam estar envolvidas na etiologia de transtornos neurocomportamentais como deficiência intelectual, esquizofrenia, transtorno bipolar e TEA [37-40]. 
No córtex cerebral normalmente os neurônios que compõem as camadas corticais são organizados em uma forma dependente da data de nascimento, na qual os neurônios nascidos tardiamente são localizados mais superficialmente que neurônios nascidos mais cedo [41-43]. Para conseguir esta disposição, os neurônios precisam migrar longos distancias desde seu local de nascimento até seu destino final, exibindo mudanças sequenciais e complexas nas suas projeções [44]. Este processo é importante tanto no desenvolvimento embrionário como no curso da sinaptogênese na vida adulta e ocorre em duas etapas basicamente: uma migração assistida pelas células gliais e outra chamada translocação somal ou migração independente da glia (Figura 2) [34,36].

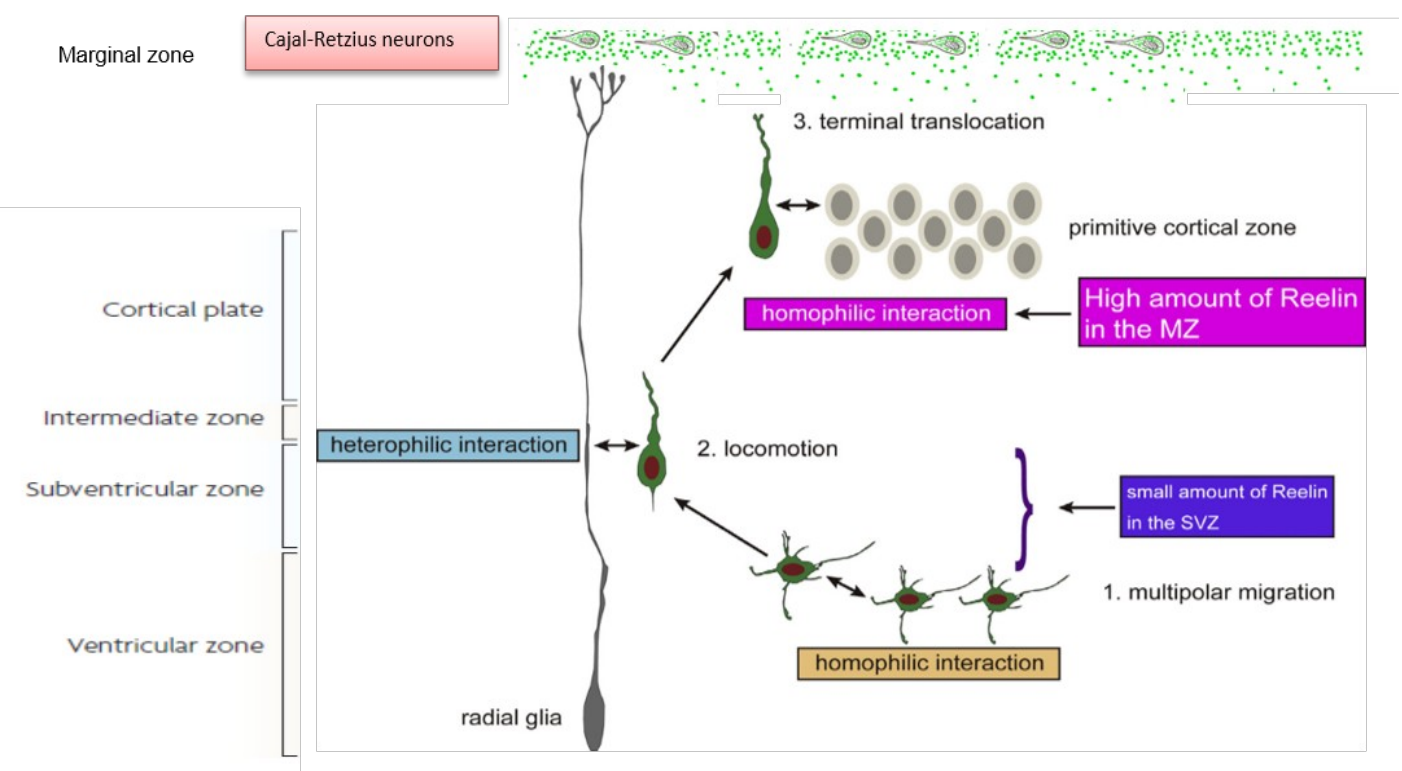

Figura 2. Fases da migração dos neurônios durante o desenvolvimento embrionário. Estrutura do córtex cerebral dos mamíferos organizado em seis camadas de células neurais. Neurônios após seu nascimento na zona ventricular (SVZ) e de sua transformação de uma morfologia multipolar para bipolar exibem um processo principal muito estável, que juntamente com fibras da glia radial (interação heterofílica) conseguem migrar longas distancias, até que o processo principal do neurônio alcance a camada mais superficial do córtex cerebral denominado zona marginal (MZ). A segunda etapa da migração, a translocação somal ou terminal, se inicia quando os corpos celulares translocam-se da fibra radial e movem-se rapidamente em direção à superfície da lamina cortical (CP), enquanto as pontas dos processos principais retêm sua ligação à $M Z$ e completam sua migração abaixo da $M Z$, atingindo sua posição final (Modificado de Sekine et al., 2014 [34]).

Os neurônios nascem na zona ventricular, iniciam a migração como células multipolares e posteriormente adquirem morfologia bipolar. Já na forma bipolar, os neurônios exibem um prolongamento principal muito estável e, dirigidos pelas fibras da glia radial, migram longas distancias até que o prolongamento principal atinja a camada mais superficial do córtex cerebral chamada de zona marginal [42,43]. Enquanto a ponta do prolongamento principal retém sua ligação à zona marginal, 
ocorre a segunda etapa da migração, na qual o corpo celular sofre translocação com o auxílio da fibra glial e move-se rapidamente em direção à superfície da lamina cortical até atingir sua posição final (Figura 2) [34,36].

Para que as camadas do córtex cerebral se formem adequadamente, uma proteína-chave envolvida no controle da migração de diversas populações neuronais é a Relina, uma grande glicoproteína da matriz extracelular. Durante o desenvolvimento cerebral, a Relina é expressa pelos neurônios Cajal-Retzius que se encontram na zona marginal do córtex cerebral e do hipocampo (Figura 2) [45]. Ela regula a transição dos neurônios da morfologia multipolar à bipolar (primeira etapa da migração), media as interações com a glia e estabiliza o processo principal dos neurônios na última etapa da migração (Figura 2) [34,36]. Finalmente quando os neurônios alcançam sua posição final, ela fornece um sinal de parada à migração [35]. No cerebelo, a Relina é produzida por células granulares na camada granular externa e promove a migração radial das células de purkinge.

$\mathrm{Na}$ idade adulta, a Relina é expressa por um subconjunto de interneurônios GABAérgicos (principais neurônios inibitórios do sistema nervoso central) no córtex e no hipocampo, e por células granulares do cerebelo [46], e parece estar envolvida na morfogênese da árvore dendrítica, sítios predominantes da neurotransmissão excitatória e da plasticidade sináptica $[47,48]$. A função essencial da Relina no estabelecimento das camadas celulares corticais é mantida após o nascimento no giro dentado do hipocampo, estrutura que participa ao longo da vida na neurogênese e na migração dos neurônios granulares no curso da sinaptogênese [35]. Assim, a Relina é importante também para os processos de aprendizagem e memória $[47,49]$.

A Relina (Figura 3) exerce suas funções ao se ligar à região extracelular dos receptores ApoER2 e VLDLR [50,51], os quais estão presentes na membrana dos neurônios migratórios.

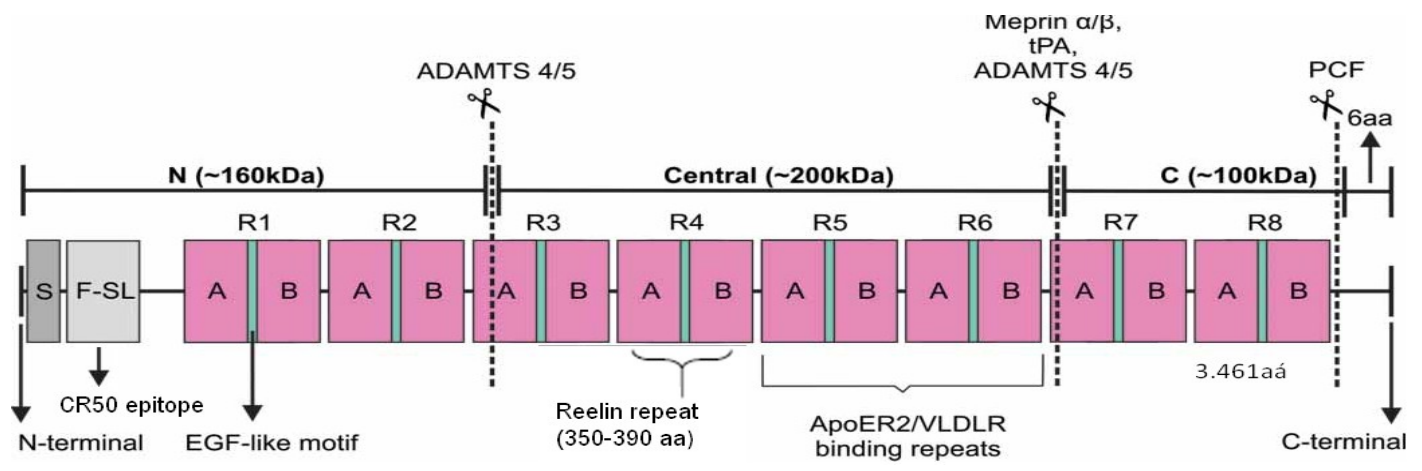


Figura 3. Estrutura da Relina e seus sítios de clivagem. A proteína Relina é codificada pelo gene RELN (NM_005045), que se localiza em humanos no cromossomo 7q22.1, contem 65 exons e abrange aproximadamente $450 \mathrm{~Kb}$ do DNA genômico. Estruturalmente é caracterizada por uma série de oito repetições consecutivas chamadas domínios da Relina (R1-8), sendo que cada repetição é composta de dois subdomínios $A$ e $B$, separadas por um padrão de cisteínas comumente encontrado em proteínas extracelulares EGF-like. A região N-terminal apresenta um peptídeo sinal (S) e um domínio semelhante a F-spondin (F-SL), que reconhecida pelo anticorpo monoclonal CR-50 é crucial para a oligomerização da proteína, a qual é necessária para seu adequado funcionamento nos processos de sinalização celular. A região C-terminal contém um fragmento rico em arginina, o qual parece desempenhar importante papel na secreção da proteína. A Relina é clivada in vivo por proteases (como ADAMTS 4/5, tPA, Meprin $\sigma / \beta$ ) em dois sítios específicos, entre as repetições 2 e 3 e 6 e 7 , gerando 3 fragmentos proteolíticos. O fragmento composto pelas repetições 3 a 6 é o único que parece ser necessário e suficiente para se ligar aos receptores de membrana ApoER2 e VLDLR, ativar diferentes cascatas de sinalização intracelular, e induzir migração de células neurais e formação das camadas corticais in vitro. Recentemente, os sítios de ligação aos receptores foram restritos às porções 5 e 6 deste fragmento [33,35] (Modificado de Santana et al., 2017 [59]).

Após a ligação da Relina a seus receptores de membrana, ocorre inicialmente a ativação da proteína adaptadora Disabled-1 (Dab1) por meio da fosforilação de resíduos específicos de tirosina pela ação das quinases da família Src (SFKs), mais especificamente Fyn e Src $[52,53]$. Essas quinases se mantém ativas por meio de um mecanismo de feedback positivo dependente da proteína Dab1 fosforilada (pDab1) $[53,54]$. Uma vez ativada, Dab1 promove então fosforilações sequenciais e ativação de vários efetores downstream, tais como a proteína adaptadora Crk, e as proteínas sinalizadoras C3G (GTP exchange factor) e Rap1 (GTPase), envolvidas na inserção de diferentes moléculas de adesão celular na membrana plasmática dos neurônios que estão migrando e que permitem interação entre esses neurônios e neurônios Cajal-Retzius produtores da Relina [55]. Ainda, varias proteínas que regulam a dinâmica do citoesqueleto e a motilidade celular, tais como Lis1 e Nck $\beta$, também são ativadas por Crk e Rap1, permitindo assim as mudanças morfológicas (multipolar para bipolar) requeridas pelos neurônios durante a migração (Figura 4) [56,57]. 


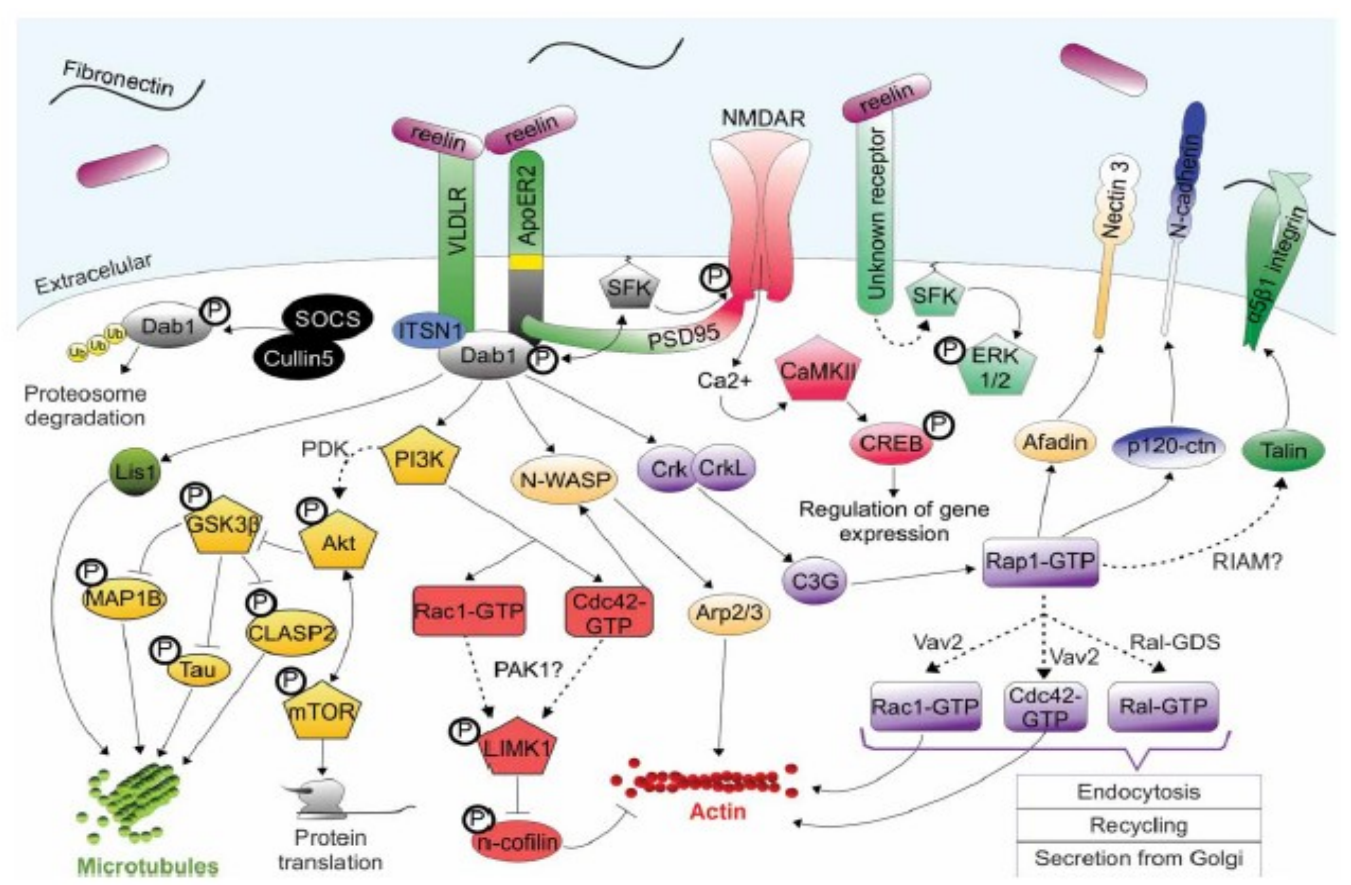

Figura 4. Via de sinalização Relina-Dab1 no desenvolvimento cerebral e na vida adulta. (Modificado de Santana et al., 2017 [59]).

Outra via que é ativada pelo eixo Relina-Dab1 é a via PI3K-Akt-mTOR (ou apenas mTOR (do inglês mammalian target of rapamycin), seja por meio da proteína adaptadora Crk que induz fosforilação de Akt, componente upstream a mTOR, ou diretamente por meio de pDab1 (Figura 4) [54,58,59]. A ativação da via mTOR após a ligação a Relina a seus receptores participa tanto da arborização dos neuritos e desenvolvimento dos dendritos [48], como da migração neuronal [60], sendo relevante para remodelamento do citoesqueleto de actina por ativar as GTPases Cdc42 e Rac1 $[59,61]$.

A duração da sinalização Relina-Dab1 é regulada pela ubiquitinação e degradação de pDab1, pFyn e pSrc por Cullin 5 (Cul5), uma E3 ubiquitina ligase $[60,62]$. É muito importante ressaltar um estudo recente e pioneiro que mostrou que o aumento excessivo da expressão da proteína Cul5 devido a hiperativação da via de sinalização mTOR (mais especificamente da via mTORC1, mTOR complex 1), leva a diminuição de pDab1 e pSFKs, ao funcionamento anormal da via Relina-Dab1, e a migração neuronal alterada no modelo animal do complexo de esclerose tuberosa (TSC) e nos tubérculos corticais de pacientes com a síndrome [60]. Assim, esse estudo mostrou pela primeira vez que existe uma regulação complexa e intercruzada entre os eixos de sinalização Relina-Dab1 e mTORC1, e que desregulação dessas sinalizações está envolvida na patofisiologia de uma forma sindrômica de TEA. 


\subsection{Variantes deletérias na Relina e transtorno do espectro do autismo não-sindrômico}

Camundongos com perda de função em ambos os alelos do gene RELN, conhecidos como reeler, apresentam um fenótipo caracterizado por tremor, ataxia, hipoplasia do cerebelo, crescimento anormal de neuritos e desorganização acentuada da citoarquitetura cerebral (incluindo laminação cortical invertida, posicionamento anormal de neurônios, orientação anormal de corpos celulares e fibras) [46]. Por outro lado, camundongos heterozigotos, embora não apresentem o mesmo fenótipo grave de tremor e ataxia, também têm algumas anormalidades comportamentais e cerebrais, como impulsividade, aprendizado associativo deficiente, diminuição da densidade dos espinhos dendríticos de neurônios do córtex cerebral e do hipocampo, e perda progressiva das células de purkinge no córtex cerebelar [63].

Em humanos, perda de função de ambos os alelos do gene RELN é incomum e é conhecida por ocasionar uma forma de lisencefalia (perda das circunvoluções do cérebro) e hipoplasia cereberal, acompanhada de retardo no desenvolvimento e epilepsia [64]. Variantes deletérias ou potencialmente deletérias em heterozigose no gene RELN têm sido descritas associadas a diferentes transtornos neurocomportamentais como esquizofrenia, epilepsia e TEA [33,46,65].

Estudos de ligação genética em famílias com pacientes com TEA desenvolvidos nas últimas décadas identificaram o locus $7 q 22$, no qual está localizado o gene RELN, como o primeiro candidato a conter genes de risco para o transtorno [66]. Estudos de associação entre variantes polimórficas presentes no gene RELN e TEA sugeriram a presença de variantes de risco neste gene [67-73]. Estudos de expressão gênica e proteica diferencial em cérebro post-mortem de pacientes com TEA relataram diminuição de expressão da Relina nos pacientes [33,46,74]. Ainda, estudos recentes de sequenciamento de exomas completos de pacientes com TEA revelam a presença de variantes pontuais potencialmente de perda de função em RELN em alguns pacientes [19,22,75-77].

Atualmente existem descritas cerca de 40 variantes raras no gene RELN identificadas em pacientes com TEA, a maior parte delas de substituição de aminoácidos em heterozigose e herdada de pais sem o transtorno [78]. Até recentemente ainda não haviam sido realizados estudos que mostrassem que essas variantes são de fato funcionais e apresentam envolvimento com TEA nos pacientes. 
Neste ano, um estudo mostrou que algumas dessas variantes (localizadas em um motivo conservado que ocorre duas vezes em cada repetição da Relina) levam a redução da secreção da proteína Relina [79]. Contudo, este estudo não explorou se o funcionamento da via de sinalização da Relina está de fato comprometido em células neurais portadoras das variantes e quais as consequências da menor secreção da Relina para o funcionamento das células [79]. Ainda, os fatos de que a maior parte das variantes encontradas no gene RELN nos pacientes com TEA são herdadas, e que pais de pacientes com lisencefalia são heterozigotos para variantes de perda de função em um dos alelos de $R E L N$, sugerem que as variantes em $R E L N$ sozinhas não sejam suficientes para causar TEA e que um segundo evento de risco, seja genético ou ambiental, seja necessário para que o transtorno se desenvolva.

\subsection{Variantes potencialmente deletérias identificadas no gene RELN em um paciente brasileiro com transtorno do espectro do autismo, hipóteses de trabalho do presente estudo e modelo experimental}

Por meio do sequenciamento completo do exoma de um grupo de pacientes brasileiros com TEA não-sindrômico, nosso grupo de pesquisa, em colaboração com o grupo da Dra. Maria Rita Passos Bueno (Instituto de Biociências da Universidade de São Paulo - IB-USP), identificou um paciente, denominado F2688, que é heterozigoto composto para variantes de substituição de aminoácido no exon 48 do gene RELN: c.C7538G:p.S2513C e c.C7634T:p.A2545V (GenBank NM_005045.3), a primeira herdada de sua mãe (heterozigota) e a segunda de seu pai (heterozigoto), os quais não apresentam TEA. Essas variantes estão presentes em frequências muito baixas $(<0,5 \%)$ nos diferentes bancos de dados populacionais, envolvem resíduos de aminoácidos conservados evolutivamente, e estão localizadas na região da Relina que se liga a seus receptores (repetição 6; Figura 3).

Ainda, cabe ressaltar que em um estudo prévio realizado por nosso grupo, identificamos que o paciente F2688 apresenta hiperativação da via de sinalização mTORC1 (de origem ainda não identificada) em células-tronco de polpa de dente decíduo (do inglês, stem cells from human exfoliated decidous teeth - SHEDs) [80]. Diversos estudos recentes têm sugerido que um importante mecanismo patofisiológico associado com a etiologia de uma parcela dos casos TEA envolve disfunção da via de sinalização mTORC1 [81]. 
Assim sendo, levando em conta as considerações discutidas nos itens anteriores, as hipóteses que orientam este trabalho são as seguintes: i) as variantes genéticas identificadas em RELN no paciente F2688 são funcionais, isto é, causam perda parcial da disfunção da via de sinalização Relina-Dab1 em células neurais, ii) existe um crosstalk anormal entre as vias de sinalização Relina-Dab1 e mTORC1 nas células neurais do paciente F2688, e o hiperfuncionamento da via mTORC1 está funcionando como um segundo evento genético que acentua a diminuição da sinalização Relina-Dab1. Para testar essas hipóteses utilizaremos como modelo experimental células-tronco pluripotentes induzidas (em inglês, induced pluripotent stem cells - iPSCs) [82] geradas a partir das SHEDs do paciente F2688, de 5 pacientes com TEA sem mutação em RELN, e de 5 indivíduos controles (pareados por sexo e idade com os pacientes). Essas células já haviam sido previamente geradas por nosso grupo de pesquisa, e já haviam sido diferenciadas com sucesso em células neuroprogenitoras (NPCs) e depois em neurônios (de acordo com protocolo descrito em [83]).

As iPSCs têm sido consideradas um modelo experimental bastante promissor para o estudo do TEA por sua capacidade de gerar tipos celulares (NPCs, neurônios, astrócitos) relevantes para o transtorno, de, quando geradas a partir de células somáticas de pacientes, preservar o background genético dos pacientes e de representar estágios iniciais do neurodesenvolvimento [84,85]. A utilização das iPSCs pode apresentar vantagens quando comparada ao uso dos modelos animais, que são limitados em recapitular transtornos humanos comportamentais complexos e multigênicos, e das amostras de cérebros post-mortem de pacientes, que geralmente permitem o estudo do cérebro em estágios mais tardios da doença [84].

Diferentes trabalhos utilizando iPSCs para estudar tanto formas sindrômicas como não-sindrômicas do TEA têm demonstrado a validade desse modelo experimental, identificando fenótipos celulares e moleculares robustos, reprodutíveis e relevantes para TEA [83,86-89]. Pode-se citar o trabalho de Griesi-Oliveira e colaboradores (2014), o qual utilizou iPSCs de um paciente com TEA não-sindrômico portador de translocação de novo balanceada que interrompe o gene TRPC6, que codifica um canal de $\mathrm{Ca}^{2+}$. Os autores mostram que as células neurais derivadas das iPSCs desse paciente apresentam alterações morfológicas (como neuritos mais curtos e menor densidade de espinhos dendríticos), funcionais (como menor formação de sinapses glutamatérgicas e menor excitabilidade), e desregulação da expressão de genes envolvidos no desenvolvimento e na função neuronal [89]. Em outro estudo, realizado com cultura neuronal tridimensional derivada de iPSCs (organoides 
cerebrais) de pacientes com TEA idiopático, Mariani e colaboradores (2015) mostraram expressão aumentada de genes relacionados com proliferação celular, diferenciação neuronal e sinaptogênese, assim como hiperprodução de neurônios inibitórios GABAérgicos (resultado do aumento na expressão do gene FOXG1) nos pacientes [90]. 


\subsection{Objetivos}

\section{Objetivos gerais:}

1. Verificar se células neuroprogenitoras derivadas do paciente F2688 apresentam disfunção da via de sinalização Relina-Dab1;

2. Verificar se células neuroprogenitoras derivadas do paciente F2688 apresentam hiperfuncionamento da via de sinalização mTORC1 como observado nas células-tronco de polpa de dente decíduo;

3. Verificar se células neuroprogenitoras derivadas do paciente F2688 apresentam crosstalk anormal entre as vias de sinalização Relina-Dab1 e mTORC1.

\section{Objetivos específicos:}

a. Verificar se células neuroprogenitoras derivadas do paciente F2688 apresentam níveis alterados de expressão do gene RELN e da proteína Relina;

b. Verificar se células neuroprogenitoras derivadas do paciente F2688 apresentam níveis alterados de expressão gênica e proteica de componentes da via de sinalização Relina-Dab1;

c. Verificar se células neuroprogenitoras derivadas do paciente F2688 apresentam níveis alterados de expressão gênica e proteica de componentes da via de sinalização mTORC1;

d. Caso seja confirmada hiperatividade da via de sinalização mTORC1 nas células neuroprogenitoras derivadas do paciente F2688, verificar se ela contribui para a disfunção do eixo de sinalização Relina-Dab1;

e. Verificar se células neuroprogenitoras derivadas do paciente F2688 apresentam migração alterada. 
CAPÍTULO 2 - CONCLUSÕES 


\section{CAPÍTULO 2 - CONCLUSÕES}

Em resumo, nossos resultados nos permitiram concluir que:

1- as variantes raras identificadas no gene $R E L N$ no paciente F2688 estão localizadas em regiões funcionalmente importantes da Relina;

2- células neuroprogenitoras derivadas de iPSCs do paciente F2688 acumulam Relina no citoplasma e apresentam menos Relina no meio extracelular;

3- células neuroprogenitoras do paciente F2688 apresentam diminuição da atividade da via de sinalização Relina-Dab1;

4- células neuroprogenitoras do paciente F2688 apresentam aumento da atividade da via de sinalização mTORC1;

5- existe uma interação alterada entre as vias de sinalização Relina-Dab1 e mTORC1 nas NPCs do paciente F2688, e o hiperfuncionamento da via mTORC1 parece contribuir para a disfunção da via Relina-Dab1 nessas células;

6- células neuroprogenitoras do paciente F2688 apresentam migração alterada. 


\section{RESUMO}

O transtorno do espectro do autismo (TEA) constitui um grupo heterogêneo e altamente prevalente de doenças do neurodesenvolvimento. Análises genômicas recentes têm revelado um grande número de variantes genéticas potencialmente deletérias nos pacientes com TEA, a maioria rara ou privada. Um enorme desafio atual é determinar quais dentre essas variantes são as que de fato estão envolvidas na etiologia do transtorno nos pacientes, e quantas variantes patogênicas são necessárias para a penetrância completa do TEA em cada paciente. Recentemente, por meio do sequenciamento completo do exoma de um subgrupo de pacientes com TEA não-sindrômico - nos quais observamos hiperfuncionamento da via de sinalização intracelular mTORC1 - identificamos que um dos pacientes (referido como F2688) é heterozigoto composto para variantes de substituição de aminoácidos raras e potencialmente deletérias no gene RELN. Este gene codifica Relina, uma grande glicoproteína de matriz extracelular que, por meio da ativação da proteína Dab1 e de diferentes vias de sinalização intracelular, controla a migração e o posicionamento dos neurônios, a arborização de neuritos, e o funcionamento das sinapses em várias regiões do encéfalo, tanto no desenvolvimento embrionário como na vida adulta. Estudos anteriores já haviam descrito variantes em heterozigose potencialmente de perda de função no gene RELN em pacientes com TEA; contudo, nenhum desses estudos investigou disfunção da sinalização Relina-Dab1 nos pacientes e, portanto, os efeitos moleculares e celulares de tais variantes sobre células neurais humanas ainda são poucos explorados. Neste trabalho, utilizando células neuroprogenitoras (NPCs) derivadas de células-tronco pluripotentes induzidas do paciente F2688, de outros pacientes com TEA sem mutação em $\operatorname{RELN}(\mathrm{n}=5)$ e de indivíduos controles $(\mathrm{n}=5)$, nós descrevemos que as NPCs do paciente F2688 apresentam: i) disfunção da via de sinalização Relina-Dab1; ii) hiperfuncionamento da via de sinalização mTORC1; iii) crosstalk anormal entre as vias de sinalização Relina-Dab1 e mTORC1, o qual é atenuado com o uso da rapamicina, um inibidor específico de mTORC1. Portanto, nossos resultados sugerem, pela primeira vez, uma relação anormal entre as vias de sinalização Relina-Dab1 e mTORC1 em TEA não-sindrômico. 


\section{ABSTRACT}

Autism Spectrum Disorder (ASD) is a heterogeneous and highly prevalent group of neurodevelopmental disorders. Whole-genome-based approaches have generated catalogues of thousands of rare and potentially deleterious genetic variants in ASD patients. However, the challenge now is to identify genuine disease-causing/risk variants among the multitude of variants discovered in each exome/genome and how many variants are required to cause the disease. Recently, we performed whole-exome sequencing in a subgroup of ASD patients - in whom we found mTORC1 signaling hyperfunction - and identified rare and potentially deleterious compound heterozygous variants in the RELN gene in one patient (called as F2688). The RELN gene encodes Reelin, a large secreted glycoprotein that controls neuronal migration, layer formation, neurite outgrowth, and plasticity of synapses in both the developing and the adult brain. Evidence from previous studies suggests that certain potential loss-of-function variants in RELN gene can contribute to ASD susceptibility; however, few studies today have directly demonstrated impairment of the Reelin signal transduction cascade in ASD patients and therefore, the molecular and cellular effects of these variants in human neuronal cells are still poorly explored. Here, by using induced pluripotent stem cells derived neuronal progenitor cells from F2688 patient, from other ASD patients who do not carry RELN disrupting variants $(n=5)$ and from control individuals $(n=5)$, we have demonstrated that F2688-derived NPCs show: i) impaired Reelin-Dab1 signaling; ii) overactive mTORC1 signaling; iii) and abnormal crosstalk between mTORC1 and Reelin-Dab1 signaling pathways, which it attenuated by rapamycin (a specific mTORC1 inhibitor). Taken together, our results point to an abnormal interplay between mTORC1 and Reelin-Dab1 networks in nonsyndromic ASD. 


\section{Referências bibliográficas referentes aos capitulos 1, 2 e 4}

1. American Psychiatric Association (2015). Characteristics, diagnosis and risk factors of Autism Spectrum Disorders.

2. Mannion, A., Leader, G., and Healy, O. (2013). An investigation of comorbid psychological disorders, sleep problems, gastrointestinal symptoms and epilepsy in children and adolescents with Autism Spectrum Disorder. Res. Autism Spectr. Disord. 7, $35-42$.

3. Mannion, A., and Leader, G. (2013). Comorbidity in autism spectrum disorder: A literature review. Res. Autism Spectr. Disord. 7, 1595-1616.

4. Romero, M., Aguilar, J.M., Del-Rey-Mejías, Á., Mayoral, F., Rapado, M., Peciña, M., Barbancho, M.A.., Ruiz-Veguilla, M., and Lara, J.P. (2016). Psychiatric comorbidities in autism spectrum disorder: A comparative study between DSM-IV-TR and DSM-5 diagnosis. Int. J. Clin. Heal. Psychol. 16, 266-275.

5. Christensen, D.L., Baio, J., Braun, Kim Van Naarden Bilder, D., Charles, J., Constantino, J., Daniels, J., Durkin, M., Fitzgerald, R.T., Kurzius-Spencer, M., Lee, L.-C., et al. (2016). Prevalence and Characteristics of Autism Spectrum Disorder Among Children Aged 8 Years - Autism and Developmental Disabilities Monitoring Network, 11 Sites, United States, 2012. MMWR Surveill. Summ. 65, 1-23.

6. Bourgeron, T. (2015). From the genetic architecture to synaptic plasticity in autism spectrum disorder. Nat. Rev. Neurosci. 16, 551-563.

7. Fombonne, E. (2009). Epidemiology of pervasive developmental disorders. Pediatr. Res. $65,591-598$.

8. Paula, C.S., Fombonne, E., Gadia, C., Tuchman, R., and Rosanoff, M. (2011). Autism in Brazil: perspectives from science and society. Rev. Assoc. Med. Bras. 57, 2-5.

9. Colvert, E., Tick, B., Mcewen, F., Stewart, C., Curran, R., Woodhouse, E., Gillan, N., Hallett, V., Lietz, S., Garnett, T., et al. (2015). Heritability of Autism Spectrum Disorder in a UK Population- Based Twin Sample. JAMA Psychiatry 72, 415-423.

10. Gaugler, T., Klei, L., Sanders, S.J., Bodea, C.A., Goldberg, A.P., Lee, A.B., Mahajan, M., Manaa, D., Pawitan, Y., Reichert, J., et al. (2014). Most genetic risk for autism resides with common variation. Nat. Genet. 46, 881-885.

11. Sandin, S., Lichtenstein, P., Larsson, H., Cm, H., and Reichenberg, a (2014). The familial risk of autism . 311, 24794370 .

12. Hallmayer, J., Cleveland, S., Torres, A., Phillips, J., Cohen, B., Torigoe, T., Miller, J., Fedele, A., Collins, J., Smith, K., et al. (2011). Genetic Heritability and Shared Environmental Factors Among Twin Pairs With Autism. Arch. Gen. Psychiatry 68, 10951102.

13. Ronald, A., and Hoekstra, R.A. (2011). Autism spectrum disorders and autistic traits: A decade of new twin studies. Am. J. Med. Genet. Part B Neuropsychiatr. Genet. 156, 255-274.

14. Richards, C., Jones, C., Groves, L., Moss, J., and Oliver, C. (2015). Prevalence of autism spectrum disorder phenomenology in genetic disorders: A systematic review and meta-analysis. The Lancet Psychiatry 2, 909-916.

15. Pinto, D., Pagnamenta, A.T., Klei, L., Anney, R., Merico, D., Regan, R., Conroy, J., 
Magalhaes, T.R., Correia, C., Brett, S., et al. (2010). Functional Impact of Global Rare Copy Number Variation in Autism Spectrum Disorder. Nature 466, 368-372.

16. Sebat, J., Lakshmi, B., Malhotra, D., Troge, J., Lese-martin, C., Walsh, T., Yamrom, B., Yoon, S., Krasnitz, A., Kendall, J., et al. (2007). Strong Association of De Novo Copy. Science (80-. ). 445, 445-449.

17. Sanders, S.J., Ercan-Sencicek, A.G., Hus, V., Luo, R., Murtha, M.T., Moreno-De-Luca, D., Chu, S.H., Moreau, M.P., Gupta, A.R., Thomson, S.A., et al. (2011). Multiple Recurrent De Novo CNVs, Including Duplications of the 7q11.23 Williams Syndrome Region, Are Strongly Associated with Autism. Neuron 70, 863-885.

18. Levy, D., Ronemus, M., Yamrom, B., Lee, Y. ha, Leotta, A., Kendall, J., Marks, S., Lakshmi, B., Pai, D., Ye, K., et al. (2011). Rare De Novo and Transmitted Copy-Number Variation in Autistic Spectrum Disorders. Neuron 70, 886-897.

19. Neale, B., Kou, Y., Liu, L., and Ma'ayan, A. (2013). Patterns and rates of exonic de novo mutations in autism spectrum disorders. Nature 485, 242-245.

20. Iossifov, I., Ronemus, M., Levy, D., Wang, Z., Hakker, I., Yamrom, B., Lee, Y., Narzisi, G., Leotta, A., Grabowska, E., et al. (2012). De novo Gene Disruptions in children on the Autistic Spectrum. Neuron 74, 285-299.

21. Simons Foundation Autism Research Initiative. Available at: http://gene.sfari.org [Accessed December 1, 2017].

22. Iossifov, I., O'Roak, B.J., Sanders, S.J., Ronemus, M., Krumm, N., Levy, D., Stessman, H.A., Witherspoon, K.T., Vives, L., Patterson, K.E., et al. (2014). The contribution of de novo coding mutations to autism spectrum disorder. Nature 515, 216-221.

23. Girirajan, S., Rosenfeld, J.A., Cooper, G.M., Antonacci, F., Siswara, P., Itsara, A., Vives, L., Walsh, T., McCarthy, S.E., Baker, C., et al. (2010). A recurrent 16p12.1 microdeletion supports a two-hit model for severe developmental delay. Nat. Genet. 42, 203-209.

24. Leblond, C.S., Heinrich, J., Delorme, R., Proepper, C., Betancur, C., Huguet, G., Konyukh, M., Chaste, P., Ey, E., Rastam, M., et al. (2012). Genetic and functional analyses of SHANK2 mutations suggest a multiple hit model of autism spectrum disorders. PLoS Genet. 8.

25. Steinberg, J., and Webber, C. (2013). The roles of FMRP-Regulated genes in autism spectrum disorder: Single- and multiple-hit genetic etiologies. Am. J. Hum. Genet. 93, 825-839.

26. Constantino, J.N. (2011). The quantitative nature of autistic social impairment. Pediatr. Res. 69, 55-62.

27. Bourgeron, T. (2016). Current knowledge on the genetics of autism and propositions for future research. C. R. Biol. 339, 300-307.

28. Sanders, S.J., He, X., Willsey, A.J., Devlin, B., Roeder, K., State, M.W., Sanders, S.J., $\mathrm{He}, \mathrm{X}$., Willsey, A.J., Ercan-sencicek, A.G., et al. (2015). Insights into Autism Spectrum Disorder Genomic Architecture and Biology from 71 Risk Loci. Neuron 87, 1215-1233.

29. Desachy, G., Croen, L.A., Torres, A.R., Kharrazi, M., Delorenze, G.N., Windham, G.C., Yoshida, C.K., and Weiss, L.A. (2015). Increased female autosomal burden of rare copy number variants in human populations and in autism families. Mol. Psychiatry 20, 170175.

30. DeRubeis, S., Pasciuto, E., Li, K.W., Fernández, E., DiMarino, D., Buzzi, A., Ostroff, L.E., Klann, E., Zwartkruis, F.J.T., Komiyama, N.H., et al. (2013). CYFIP1 coordinates mRNA translation and cytoskeleton remodeling to ensure proper dendritic Spine formation. Neuron 79, 1169-1182. 
31. Gilman, S.R., lossifov, I., Levy, D., Ronemus, M., Wigler, M., and Vitkup, D. (2011). Rare De Novo Variants Associated with Autism Implicate a Large Functional Network of Genes Involved in Formation and Function of Synapses. Neuron 70, 898-907.

32. Pinto, D., Delaby, E., Merico, D., Barbosa, M., Merikangas, A., Klei, L., Thiruvahindrapuram, B., Xu, X., Ziman, R., Wang, Z., et al. (2014). Convergence of Genes and Cellular Pathways Dysregulated in Autism Spectrum Disorders. Am. J. Hum. Genet. 94, 677-694.

33. Fatemi, S.H. (2005). Reelin glycoprotein: Structure, biology and roles in health and disease. Reel. Glycoprotein Struct. Biol. Roles Heal. Dis. 23, 1-440.

34. Sekine, K., Kubo, K.I., and Nakajima, K. (2014). How does Reelin control neuronal migration and layer formation in the developing mammalian neocortex? Neurosci. Res. $86,50-58$.

35. D'Arcangelo, G. (2014). Reelin in the Years: Controlling Neuronal Migration and Maturation in the Mammalian Brain. Adv. Neurosci. 2014, 1-19.

36. Förster, E. (2014). Reelin, neuronal polarity and process orientation of cortical neurons. Neuroscience 269C, 102-111.

37. Guerrini, R. (2005). Genetic malformations of the cerebral cortex and epilepsy. Epilepsia $46,32-37$.

38. Guerrini, R., and Parrini, E. (2010). Neuronal migration disorders. Neurobiol. Dis. 38, 154-166.

39. Yang, Y., Fung, S.J., Rothwell, A., Tianmei, S., and Weickert, C.S. (2011). Increased interstitial white matter neuron density in the dorsolateral prefrontal cortex of people with schizophrenia. Biol. Psychiatry 69, 63-70.

40. Wegiel, J., Kuchna, I., Nowicki, K., Imaki, H., Wegiel, J., Marchi, E., Ma, S.Y., Chauhan, A., Chauhan, V., Bobrowicz, T.W., et al. (2010). The neuropathology of autism: Defects of neurogenesis and neuronal migration, and dysplastic changes. Acta Neuropathol. 119, 755-770.

41. Bystron, I., Blakemore, C., and Rakic, P. (2008). Development of the human cerebral cortex: Boulder Committee revisited. Nat. Rev. Neurosci. 9, 110-122.

42. Nadarajah, B., Alifragis, P., Wong, R.O.L., and Parnavelas, J.G. (2003). Neuronal Migration in the Developing Cerebral Cortex: Observations Based on Real-time Imaging. 607-611.

43. Cooper, J.A. (2008). A mechanism for inside-out lamination in the neocortex. Trends Neurosci. 31, 113-119.

44. Leong, S.Y., and Turnley, A.M. (2011). Regulation of adult neural precursor cell migration. Neurochem. Int. 59, 382-393.

45. Hirota, Y., and Nakajima, K. (2017). Control of Neuronal Migration and Aggregation by Reelin Signaling in the Developing Cerebral Cortex. Front. Cell Dev. Biol. 5, 1-8.

46. Fatemi, S.H. (2001). Reelin mutations in mouse and man: from reeler mouse to schizophrenia, mood disorders, autism and lissencephaly. Mol. Psychiatry 6, 129-133.

47. Lakatosova, S., and Ostatnikova, D. (2012). Reelin and its complex involvement in brain development and function. Int. J. Biochem. Cell Biol. 44, 1501-1504.

48. Jossin, Y., and Goffinet, A.M. (2007). Reelin Signals through Phosphatidylinositol 3Kinase and Akt To Control Cortical Development and through mTor To Regulate Dendritic Growth. Mol. Cell. Biol. 27, 7113-7124. 
49. Folsom, T.D., and Fatemi, S.H. (2013). The involvement of Reelin in neurodevelopmental disorders. Neuropharmacology 68, 122-135.

50. Hiesberger, T., Trommsdorff, M., Howell, B.W., Goffinet, A., Mumby, M.C., Cooper, J.A., and Herz, J. (1999). Direct binding of Reelin to VLDL receptor and ApoE receptor 2 induces tyrosine phosphorylation of Disabled-1 and modulates tau phosphorylation. Neuron 24, 481-489.

51. Strasser, V., Fasching, D., Hauser, C., Mayer, H., Bock, H.H., Hiesberger, T., Herz, J., Weeber, E.J., Sweatt, J.D., Pramatarova, A., et al. (2004). Receptor clustering is involved in Reelin signaling. Mol. Cell. Biol. 24, 1378-86.

52. Arnaud, L., Ballif, B.A., Förster, E., and Cooper, J.A. (2003). Fyn tyrosine kinase is a critical regulator of Disabled-1 during brain development. Curr. Biol. 13, 9-17.

53. Bock, H.H., and Herz, J. (2003). Reelin Activates Src Family Tyrosine Kinases in Neurons. Curr. Biol. 13, 18-26.

54. Lee, G.H., and D'Arcangelo, G. (2016). New Insights into Reelin-Mediated Signaling Pathways. Front. Cell. Neurosci. 10, 1-8.

55. Jossin, Y., and Cooper, J. (2011). Reelin, Rap1 and N-cadherin orient the migration of multipolar neurons in the developing neocortex. Nat. Neurosci. 14, 697-703.

56. Assadi, A.H., Zhang, G., Beffert, U., McNeil, R.S., Renfro, A.L., Niu, S., Quattrocchi, C.C., Antalffy, B.A., Sheldon, M., Armstrong, D.D., et al. (2003). Interaction of reelin signaling and Lis1 in brain development. Nat. Genet. 35, 270-276.

57. Pramatarova, A., Ochalski, P.G., Chen, K., Gropman, A., Myers, S., Min, K.T., and Howell, B.W. (2003). Nck beta interacts with tyrosine-phosphorylated disabled 1 and redistributes in Reelin-stimulated neurons. Mol Cell Biol 23, 7210-7221.

58. Stranahan, A.M., Erion, J.R., and Wosiski-Kuhn, M. (2013). Reelin signaling in development, maintenance, and plasticity of neural networks. Ageing Res. Rev. 12, 815822.

59. Santana, J., and Marzolo, M.-P. (2017). The functions of Reelin in membrane trafficking and cytoskeletal dynamics: implications for neuronal migration, polarization and differentiation. Biochem. J. 474, 3137-3165.

60. Moon, Park, Park, Cho, Hughes, McKenna, J., Goetzl, L., Cho, S.H., Crino, P.B., Gambello, M.J., et al. (2015). Impaired Reelin-Dab1 signaling contributes to neuronal migration deficits of tuberous sclerosis complex. Cell Rep. 12, 965-978.

61. Liu, L., Luo, Y., Chen, L., Shen, T., Xu, B., Chen, W., Zhou, H., Han, X., and Huang, S. (2010). Rapamycin inhibits cytoskeleton reorganization and cell motility by suppressing RhoA expression and activity. J. Biol. Chem. 285, 38362-38373.

62. Feng, L., Allen, N.S., Simo, S., and Cooper, J. a. (2007). Cullin 5 regulates Dab1 protein levels and neuron positioning during cortical development. Genes Dev. 21, 2717-2730.

63. Qiu, S., Korwek, K.M., Pratt-Davis, A.R., Peters, M., Bergman, M.Y., and Weeber, E.J. (2006). Cognitive disruption and altered hippocampus synaptic function in Reelin haploinsufficient mice. Neurobiol. Learn. Mem. 85, 228-242.

64. Hong, S.E., Shugart, Y.Y., Huang, D.T., Al Shahwan, S., Grant, P.E., Hourihane, J.O.B., Martin, N.D.T., and Walsh, C.A. (2000). Autosomal recessive lissencephaly with cerebellar hypoplasia is associated with human RELN mutations. Nat. Genet. 26, 93-96.

65. Dazzo, E., Fanciulli, M., Serioli, E., Minervini, G., Pulitano, P., Binelli, S., Di Bonaventura, C., Luisi, C., Pasini, E., Striano, S., et al. (2015). Heterozygous reelin mutations cause autosomal-dominant lateral temporal epilepsy. Am. J. Hum. Genet. 96, 992-1000. 
66. IMGSAC, I.M.G.S. of A. (1998). A full genome screen for autism with evidence for linkage to a region on chromosome 7q. Hum. Mol. Genet. 7, 571-578.

67. Persico, A.M., D'agruma, L., Maiorano, N., Totaro, A., Militerni, R., Bravaccio, C., Wassink, T.H., Schneider, C., Melmed, R., Trillo, S., et al. (2001). Reelin gene alleles and haplotypes as a factor predisposing to autistic disorder. Mol. Psychiatry 6, 150-159.

68. Serajee, F.J., Zhong, H., and Mahbubul Huq, A.H.M. (2006). Association of Reelin gene polymorphisms with autism. Genomics $87,75-83$.

69. Holt, R., Barnby, G., Maestrini, E., Bacchelli, E., Brocklebank, D., Sousa, I., Mulder, E.J., Kantojärvi, K., Järvelä, I., Klauck, S.M., et al. (2010). Linkage and candidate gene studies of autism spectrum disorders in European populations. Eur. J. Hum. Genet. 18, 1013-1020.

70. Fu, X., Mei, Z., and Sun, L. (2013). Association between the g.296596G > A genetic variant of RELN gene and susceptibility to autism in a Chinese Han population. Genet. Mol. Biol. 36, 486-489.

71. Wang, Z., Hong, Y., Zou, L., Zhong, R., Zhu, B., Shen, N., Chen, W., Lou, J., Ke, J., Zhang, T., et al. (2014). Reelin gene variants and risk of autism spectrum disorders: An integrated meta-analysis. Am. J. Med. Genet. Part B Neuropsychiatr. Genet. 165, 192200.

72. Ashley-Koch, A.E., Jaworski, J., Ma, D.Q., Mei, H., Ritchie, M.D., Skaar, D.A., Robert Delong, G., Worley, G., Abramson, R.K., Wright, H.H., et al. (2007). Investigation of potential gene-gene interactions between APOE and RELN contributing to autism risk. Psychiatr. Genet. 17, 221-226.

73. Li, H., Li, Y., Shao, J., Li, R., Qin, Y., Xie, C., and Zhao, Z. (2008). The association analysis of RELN and GRM8 genes with autistic spectrum disorder in Chinese Han population. Am. J. Med. Genet. Part B Neuropsychiatr. Genet. 147, 194-200.

74. Garbett, K., Ebert, P.J., Mitchell, A., Lintas, C., Manzi, B., Mirnics, K., and Persico, A.M. (2008). Immune transcriptome alterations in the temporal cortex of subjects with autism. Neurobiol. Dis. 30, 303-311.

75. De Rubeis, S., He, X., Goldberg, A.P., Poultney, C.S., Samocha, K., Ercument Cicek, A., Kou, Y., Liu, L., Fromer, M., Walker, S., et al. (2014). Synaptic, transcriptional and chromatin genes disrupted in autism. Nature 515, 209-215.

76. Yuen, R.K.C., Thiruvahindrapuram, B., Merico, D., Walker, S., Tammimies, K., Hoang, N., Chrysler, C., Nalpathamkalam, T., Pellecchia, G., Liu, Y., et al. (2015). Wholegenome sequencing of quartet families with autism spectrum disorder. Nat. Med. 21, 185-191.

77. Stessman, H.A.F., Xiong, B., Coe, B.P., Wang, T., Hoekzema, K., Fenckova, M., Kvarnung, M., Gerdts, J., Trinh, S., Cosemans, N., et al. (2017). Targeted sequencing identifies 91 neurodevelopmental-disorder risk genes with autism and developmentaldisability biases. Nat. Genet. 49, 515-526.

78. Lammert, D.B., and Howell, B.W. (2016). RELN Mutations in Autism Spectrum Disorder. Front. Cell. Neurosci. 10, 84.

79. Lammert, D.B., Middleton, F.A., Pan, J., Olson, E.C., and Howell, B.W. (2017). The de novo autism spectrum disorder RELN R2290C mutation reduces Reelin secretion and increases protein disulfide isomerase expression. J. Neurochem. 142, 89-102.

80. Suzuki, A.M., Griesi-Oliveira, K., de Oliveira Freitas Machado, C., Vadasz, E., Zachi, E.C., Passos-Bueno, M.R., and Sertie, A.L. (2015). Altered mTORC1 signaling in multipotent stem cells from nearly $25 \%$ of patients with nonsyndromic autism spectrum disorders. Mol. Psychiatry 20, 551-552. 
81. Magdalon, J., Sánchez-Sánchez, S.M., Griesi-Oliveira, K., and Sertié, A.L. (2017). Dysfunctional mTORC1 signaling: A convergent mechanism between syndromic and nonsyndromic forms of autism spectrum disorder? Int. J. Mol. Sci. 18, 1-27.

82. Takahashi, K., Tanabe, K., Ohnuki, M., Narita, M., Ichisaka, T., Tomoda, K., and Yamanaka, S. (2007). Induction of Pluripotent Stem Cells from Adult Human Fibroblasts by Defined Factors. Cell 131, 861-872.

83. Marchetto, M.C.N.M., Carromeu, C., Acab, A., Yu, D., Yeo, G.W., Mu, Y., Chen, G., Gage, F.H., and Muotri, A.R. (2010). A model for neural development and treatment of Rett syndrome using human induced pluripotent stem cells. Cell 143, 527-539.

84. Beltrão-Braga, P.C.B., and Muotri, A.R. (2016). Modeling autism spectrum disorders with human neurons. Brain Res. 1656, 1-8.

85. Takahashi, K., and Yamanaka, S. (2006). Induction of Pluripotent Stem Cells from Mouse Embryonic and Adult Fibroblast Cultures by Defined Factors. Cell 126, 663-676.

86. Urbach, A., Bar-Nur, O., Daley, G.Q., and Benvenisty, N. (2010). Differential modeling of fragile $X$ syndrome by human embryonic stem cells and induced pluripotent stem cells. Cell Stem Cell 6, 407-411.

87. Amenduni, M., De Filippis, R., Cheung, A.Y.L., Disciglio, V., Epistolato, M.C., Ariani, F., Mari, F., Mencarelli, M.A., Hayek, Y., Renieri, A., et al. (2011). IPS cells to model CDKL5related disorders. Eur. J. Hum. Genet. 19, 1246-1255.

88. Paşca, S.P., Portmann, T., Voineagu, I., Yazawa, M., Shcheglovitov, A., Paşca, A.M., Cord, B., Palmer, T.D., Chikahisa, S., Nishino, S., et al. (2011). Using iPSC-derived neurons to uncover cellular phenotypes associated with Timothy syndrome. Nat. Med. $17,1657-1662$.

89. Griesi-Oliveira, K., Acab, A., Gupta, A.R., Sunaga, D.Y., Chailangkarn, T., Nicol, X., Nunez, Y., Walker, M.F., Murdoch, J.D., Sanders, S.J., et al. (2014). Modeling nonsyndromic autism and the impact of TRPC6 disruption in human neurons. Mol. Psychiatry 20, 1350-1365.

90. Mariani, J., Coppola, G., Zhang, P., Abyzov, A., Provini, L., Tomasini, L., Amenduni, M., Szekely, A., Palejev, D., Wilson, M., et al. (2015). FOXG1-Dependent Dysregulation of GABA/Glutamate Neuron Differentiation in Autism Spectrum Disorders. Cell 162, 375390.

91. Bordoli, L., Kiefer, F., Arnold, K., Benkert, P., Battey, J., and Schwede, T. (2009). Protein structure homology modeling using SWISS-MODEL workspace. Nat. Protoc. 4, 1-13.

92. Hirai, H., Yasui, N., Yamashita, K., Tabata, S., Yamamoto, M., Takagi, J., and Nogi, T. (2017). Structural basis for ligand capture and release by the endocytic receptor ApoER2. EMBO Rep. 18, 982-999.

93. DeLano, W. (2002). Pymol: An open-source molecular graphics tool. CCP4 Newsl. Protein Crystallogr.

94. Laplante, M., and Sabatini, D.M. (2012). mTOR Signaling in Growth Control and Disease. Cell 149, 274-293.

95. Sheldon, M., Rice, D.S., D’Arcangelo, G., Yoneshima, H., Nakajima, K., Mikoshiba, K., Howell, B.W., Cooper, J.A., Goldowitz, D., and Curran, T. (1997). Scrambler and yotari disrupt the disabled gene and produce a reeler- like phenotype in mice. Nature 389, 730-733.

96. Rice, D.S., Sheldon, M., D'Arcangelo, G., Nakajima, K., Goldowitz, D., and Curran, T. (1998). Disabled-1 acts downstream of Reelin in a signaling pathway that controls laminar organization in the mammalian brain. Development 125, 3719-3729. 
97. Trommsdorff, M., Gotthardt, M., Hiesberger, T., Shelton, J., Stockinger, W., Nimpf, J., Hammer, R.E., Richardson, J.A., and Herz, J. (1999). Reeler/disabled-like disruption of neuronal migration in knockout mice lacking the VLDL receptor and ApoE receptor 2. Cell 97, 689-701.

98. Kuo, G. (2005). Absence of Fyn and Src Causes a Reeler-Like Phenotype. J. Neurosci. 25, 8578-8586.

99. Bock, H.H., Jossin, Y., May, P., Bergner, O., and Herz, J. (2004). Apolipoprotein E receptors are required for reelin-induced proteasomal degradation of the neuronal adaptor protein disabled-1. J. Biol. Chem. 279, 33471-33479.

100. Splawski, I., Yoo, D.S., Stotz, S.C., Cherry, A., Clapham, D.E., and Keating, M.T. (2006). CACNA1H mutations in autism spectrum disorders. J. Biol. Chem. 281, 22085-22091.

101. Napoli, I., Mercaldo, V., Boyl, P.P., Eleuteri, B., Zalfa, F., De Rubeis, S., Di Marino, D., Mohr, E., Massimi, M., Falconi, M., et al. (2008). The Fragile X Syndrome Protein Represses Activity-Dependent Translation through CYFIP1, a New 4E-BP. Cell 134, 1042-1054.

102. Oguro-Ando, A., Rosensweig, C., Herman, E., Nishimura, Y., Werling, D., Bill, B.R., Berg, J.M., Gao, F., Coppola, G., Abrahams, B.S., et al. (2014). Increased CYFIP1 dosage alters cellular and dendritic morphology and dysregulates mTOR. Mol. Psychiatry 20, 110. 